\title{
THE TOROIDAL PUMP LIMITER ALT-II IN TEXTOR
}

\author{
K. H. FINKEN, ${ }^{*} \dagger$ D. REITER,$\dagger$ T. DENNER,$\dagger$ K. H. DIPPEL,$\dagger$ J. HOBIRK, $\dagger$ G. MANK, $\dagger$ \\ H. KEVER,$\dagger$ G. H. WOLF $\uparrow$ N. NODA, $\ddagger$ A. MIYAHARA $\neq$ T. SHOJI,${ }^{\S}$ K. N. SATO, $\ddagger$ K. AKAISHI, $\ddagger$ \\ J. A. BOEDO," J. N. BROOKS, ${ }^{*}$ R. W. CONN," W. J. CORBETT," R. P. DOERNER, " D. GOEBEL"\| \\ D. S. GRAY, D. L. HILLIS, ** J. HOGAN, ** R. T. McGRATH, $\dagger \dagger$ M. MATSUNAGA, $\neq+$ \\ R. MOYER, $\|$ R. E. NYGREN, $\dagger \dagger$ and J. WATKINS $\dagger \dagger$ \\ $\dagger$ Institut für Plasmaphysik, Forschungszentrum Jülich GmbH, EURATOM Association, Trilateral Euregio Cluster, \\ D-52425 Jülich, Germany \\ $\ddagger$ National Institute for Fusion Science, Orochi-Cho, Toki, Gifu, Japan \\ ${ }^{\S}$ Nagoya University, Department of Energy Engineering and Science, 464-8603 Nagoya, Japan \\ "University of California, San Diego, Department of Applied Mechanics and Engineering Sciences \\ San Diego, California \\ \#Argonne National Laboratory, Argonne, Illinois \\ ** Oak Ridge National Laboratory, Building 9201-2, MS-8072, Oak Ridge, Tennessee \\ $\dagger \dagger$ Sandia National Laboratories, Albuquerque, New Mexico \\ \$Toyama University, Hydrogen Isotope Research Center, Gofuku 3190, Toyama 930-8555, Japan
}

Received April 29, 2004

Accepted for Publication June 18, 2004

The Advanced Limiter Test (ALT) project is the focus of a fruitful and intense International Energy Agreement collaboration on TEXTOR. The pump limiter is a mechanical boundary that is laid out for taking the full heat load of TEXTOR, namely $8 \mathrm{MW}$ (assuming $2 \mathrm{MW}$ radiated power) for $10 \mathrm{~s}$, and provides a pumping efficiency of at least $5 \%$ of the working gas. This layout is adopted from the requirements of a fusion reactor: It is mandatory to remove both the full power that is convected to the limiter or divertor and the helium ash that is generated in the fusion process. In order to obtain pumping for all gases, the ALT-II is equipped with turbomolecular pumps.
A short description of ALT-II is given, and the power and particle fluxes to the limiter surface and into the exhaust scoops are discussed. Requirements of the helium removal rate for a reactor and relevant measurements are discussed, and particle removal and the power distribution to the limiters are treated. Related topics of the ALT-II program were hydrogen recycling and the measurement of turbulence-induced anomalous particle transport in the plasma edge.

KEYWORDS: pump limiter, particle exhaust, power exhaust

\section{INTRODUCTION}

Over many years, the Advanced Limiter Test (ALT) project was the focus of a fruitful and intense International Energy Agency (IEA) collaboration on TEXTOR. The engineering part of the work was primarily conducted by the groups of Sandia National Laboratories (United States), National Institute for Fusion Science (NIFS)/Nagoya (Japan), and Forschungszentum Jülich (FZJ) (Germany), while the physics exploitation was led by the groups of University of California, Los Angeles/ University of California, San Diego, Oak Ridge National

\footnotetext{
*E-mail: k.h.finken@fz-juelich.de
}

Laboratory (United States), NIFS/University of Nagoya (Japan), and of the FZJ. For concept testing, at first the modular pump limiter ALT-I (Refs. 1 through 10) (1983 to 1987) was installed on TEXTOR followed by the final version ALT-II (from 1988 on).

The pump limiter ALT-II (Refs. 11 through 19) is a mechanical boundary that is laid out for taking the full heat load of TEXTOR, namely $8 \mathrm{MW}$ (assuming $2 \mathrm{MW}$ radiated power) for $10 \mathrm{~s}$, and provides a pumping efficiency of at least $5 \%$ of the working gas. These layout characteristics are adopted from the requirements of a fusion reactor: It is mandatory to remove both the full power that is convected to the limiter or divertor and the helium ash that is generated in the fusion process. In 
order to obtain pumping for all gases, the ALT-II is equipped with turbomolecular pumps. Cryopumps or $\mathrm{ZrAl}$ getter pumps may have considerably higher pumping speed, and they can be located closer to the plasma; they have, however, a limited use for helium removal studies.

Pump limiters have received less attention in large tokamak programs than magnetic divertors because the plasma-limiter surface is in close proximity to the plasma core and because high confinement scenarios are less easily obtained in major machines with this configuration. In contrast, limiters require no additional magnets and may permit better use of space than divertors. These are issues potentially important for future tokamak fusion reactors. The aim of the ALT-II program was to establish a database for large area pump limiters operating in high power tokamaks to determine the physics of particle exhaust and to optimize the performance of the core plasma with the pumped toroidal limiter system. This goal was indeed successful by achieving the high confinement radiative improved (RI) mode. Related topics of material performance ${ }^{20}$ and power removal were investigated as well.

In the following sections, a short description of ALT-II is given followed by a discussion of the power and particle fluxes to the limiter surface and into the exhaust scoops. Then, the requirements of the helium removal rate for a reactor and relevant measurements are discussed. After Sec. I, general particle removal and the power distribution to the limiters are treated. Related topics of the ALT-II program were hydrogen recycling and the measurement of turbulence-induced anomalous particle transport in the plasma edge.

\section{THE PUMP LIMITER DESIGN}

The ALT-II consists essentially of eight blades located at the low field side (LFS), 45 deg below the equatorial plane. The major fraction of the power and particle flow is intercepted by a relatively thin blade. Each blade spans $28 \mathrm{~cm}$ poloidally and $143 \mathrm{~cm}$ toroidally and is radially adjustable by jacks attached near the blade tips. The base plates are constructed from INCONEL ${ }^{\circledR} 625$ plate material conformed with compound toroidal and poloidal curvature, with 28 graphite tiles bolted on the plasma-facing side. Three thicknesses of the blade including the graphite tiles have been selected, namely, $12 \mathrm{~mm}$ for highest particle removal, $17 \mathrm{~mm}$ as "standard" configuration, and $20 \mathrm{~mm}$ after the flux swing increase of TEXTOR in order to cope with a conductive/convective power flux of $6 \mathrm{MW}$ for $10 \mathrm{~s}$. The first two sets of graphite tiles have tile curvatures in the poloidal direction only while the latter type is curved also toroidally. The backs of the base plates are protected from the plasma contact by a $2-\mathrm{mm}$ tile overlap. Figure 1a shows a photograph of
ALT-II taken from the TEXTOR chamber, and Fig. 1b shows a cross section of the pumping device.

For the construction of a pump limiter one takes advantage of the fact that the decay lengths of power and density are not equal. If $\lambda_{n}$ and $\lambda_{T}$ are the decay lengths for density and temperature in the scrape-off layer (SOL), then the particle flux is given by $\Gamma=n c_{s}$ leading to $\lambda_{\Gamma}=$ $2 \lambda_{n} \lambda_{T} /\left(2 \lambda_{T}+\lambda_{n}\right)$ and the power flux by $q=k T n c_{s}$ resulting in $\lambda_{q}=2 \lambda_{T} \lambda_{n} /\left(2 \lambda_{T}+3 \lambda_{n}\right)$. Experimentally, an exponential decay length ratio of $\lambda_{T} / \lambda_{n}=2$ is observed, and therefore $\lambda_{\Gamma} / \lambda_{q}=7 / 5$ is measured, i.e., the particle flux layer is wider than the power flux. Measured data with the TEXTOR ALT-II configuration are $\lambda_{n}=$ $14 \mathrm{~mm}, \lambda_{T}=28 \mathrm{~mm}, \lambda_{\Gamma}=11.2 \mathrm{~mm}$, and $\lambda_{q}=8 \mathrm{~mm}$.

The decay length for particles is wider than that for power. Much of the plasma flow behind the limiter blade enters one of the eight pump limiter scoops. At the end of the scoop the incoming ions are neutralized and scattered into the pumping duct. These particles are either pumped by turbopumps (pumping speed 8 times $3000 \ell / \mathrm{s}$ ) or escape back through the front opening of the scoop to the plasma. The calculated exhaust efficiency is $8 \%$ assuming that $50 \%$ of the particles hitting the neutralizer plate are pumped.

\section{HELIUM REMOVAL}

\section{III.A. Basic Considerations}

In a fusion reactor, the steady-state burn energy criterion is obtained from balancing the energy gain of newly born $\alpha$ particles with the losses due to radiation, convection, and diffusion. An additional burn criterion is obtained by including the particle balance, i.e., by adding the creation rate of the helium due to fusion and its loss due to helium exhaust. ${ }^{21,22}$ In contrast to the conventional burn criterion, one obtains closed curves in the space given by the product $n T \tau_{E}$ and by the temperature. These curves are the curves of existence of a stationary burning fusion plasma as shown in Fig. 2. The parameter in the figure is the ratio $\rho$ of the helium residence time $\tau_{\mathrm{He}}^{*}$ to the energy confinement time $\tau_{E}: \rho=\tau_{\mathrm{He}}^{*} / \tau_{E}$ in the discharge vessel. A stationary fusion process is only possible if the ratio $\rho$ is smaller than about $\rho \approx 14$. This is a strong requirement on the exhaust capability of a fusion reactor, and it is valid in addition only for a clean D-T plasma. If additional impurities are present, the ratio $\rho$ becomes increasingly more severe.

\section{III.B. Experimental Results}

Because ALT-II was equipped with turbomolecular pumps, TEXTOR was the first device where active helium removal could be studied. Helium was injected in the stationary phase of the discharge as a short puff 


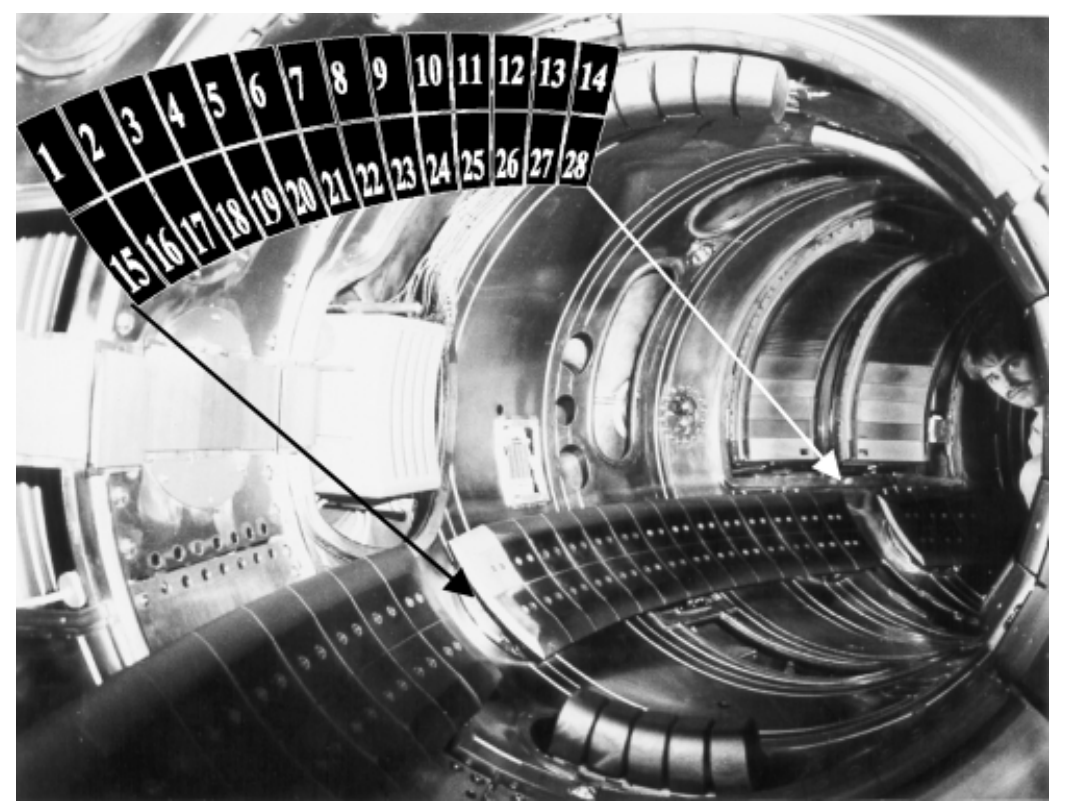

(a)

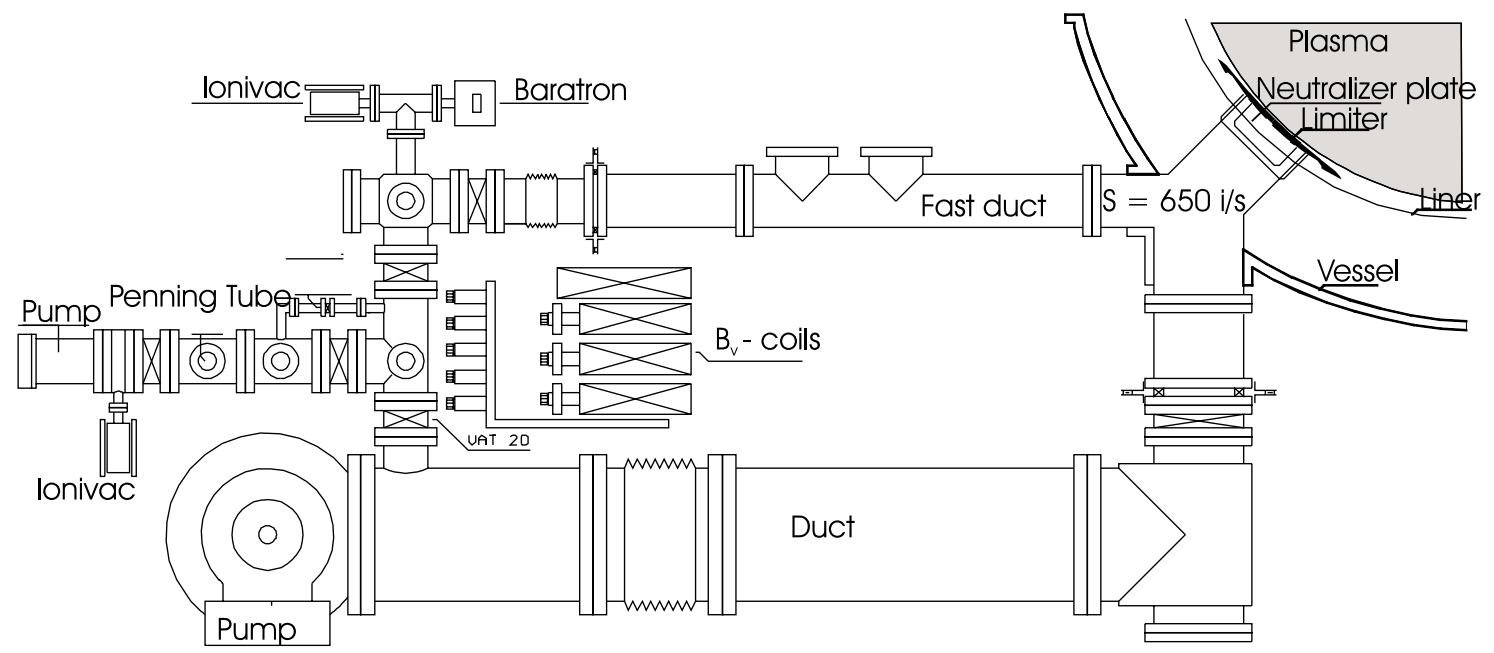

(b)

Fig. 1. (a) Photo of the interior of TEXTOR with the ALT-II toroidal pump limiter and (b) schematic cross section of the ALT-II pumping port.

leading to a helium concentration in the discharge of $\sim 10 \%$. It was found that-in contrast to many other gases-injected helium has a high fueling efficiency and that the sticking probability on the walls for helium is very low. ${ }^{23}$. The helium and deuterium densities inside the discharge are measured by charge exchange spectroscopy, the recycling helium at the limiter surface by emission spectroscopy and pumped helium complementarily by a Penning (see Sec. III.F) in the exhaust line. Figure 3 shows the time dependence of the electron density and helium in the discharge with the number of activated ALT-II pumps as a parameter. ${ }^{24-26}$ One sees that the electron density increases transiently at the onset of neutral beam injection (NBI) and at the time of the He puff; soon it stabilizes at the preset value. The helium density remains constant if the pumps are switched off and decays with activated pumping. The recycling of helium has been found to drop from 1 (no pumping) to 0.92 with full pumping; this corresponds to a maximum exhaust efficiency of $8 \%$ for helium.

As already pointed out, the critical parameter for a fusion reactor is the ratio of the helium residence time in the plasma to the energy confinement time $\rho=\tau_{\mathrm{He}}^{*} / \tau_{E}$. For this specific discharge, the measurement yields $\tau_{\mathrm{He}}^{*}=$ $0.62 \mathrm{~s}, \tau_{E}=30 \mathrm{~ms} \rightarrow \rho=20$; however, values of $\rho$ below 10 are possible with the pump limiter, in particular for high density RI-mode discharges. ${ }^{27}$ Divertor tokamaks report exhaust efficiencies that are slightly higher. ${ }^{28}$ 


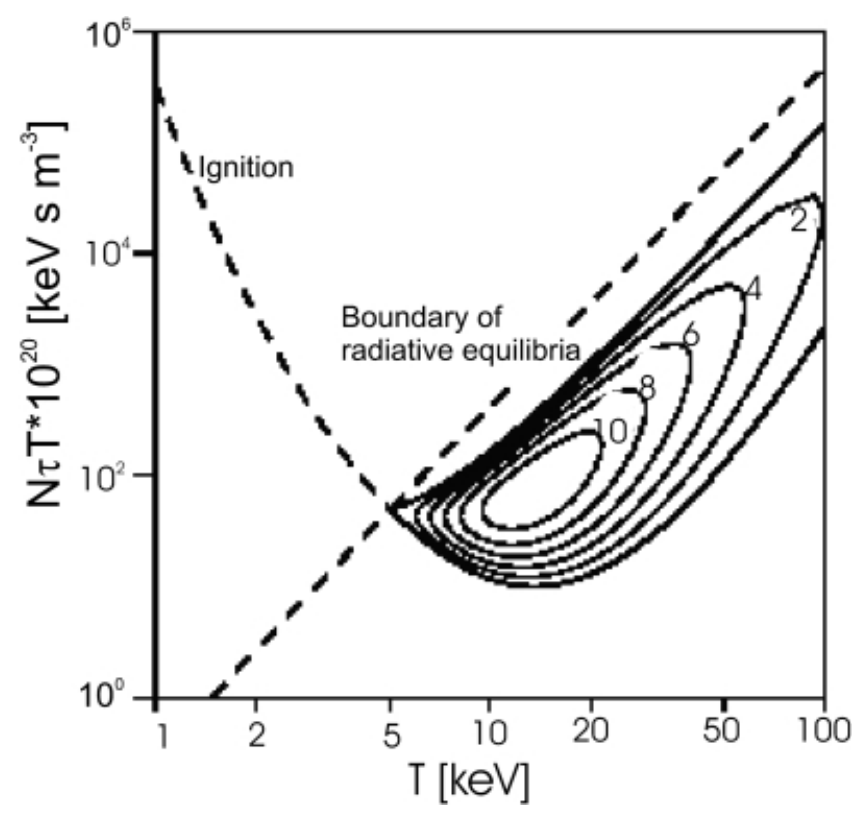

Fig. 2. Curves $n_{e} \tau_{E} T=f(T)$ for nonideal ignited equilibria, radiation limit, and boundary of radiative equilibria assuming an impurity concentration of $2 \%$ beryllium.

\section{III.C. Ion Cyclotron Resonance Heating Interaction with $\alpha$ Particles}

According to a proposal of C. S. Chang ${ }^{29-31}$ resonant ion cyclotron waves interacting off-axis with slowing down $\alpha$ particles could be utilized to expel the alphas from the discharge. The mechanism is based on a neoclassical effect and requires either a directed launching of the ion cyclotron waves or a different population of the energetic He ions in co- and counterdirections. On TEXTOR, we have been using the velocity anisotropy by injecting $50-\mathrm{keV}^{3} \mathrm{He}$ ions with a neutral beam injector; the ${ }^{3} \mathrm{He}$ isotope was selected to facilitate the ion cyclotron resonance with the fast ions while avoiding the resonance with the background deuterium. The heating acts on passing particles. Let us assume that we have predominantly copassing ${ }^{3} \mathrm{He}$ that for the given field configuration moves helically upward at the LFS [and downwards at the high field side (HFS)]; this movement is superimposed on the $\nabla B$ drift showing upward. If the ${ }^{3} \mathrm{He}$ is heated at the LFS, the drift excursion is increased if the particles arrive at the top of the machine. On their way down, the ${ }^{3} \mathrm{He}$ loses energy again by the drag force, and therefore, the enhanced orbit excursion on top is not compensated at the bottom. On average the ${ }^{3} \mathrm{He}$ makes an outward drift. One can easily see that the average drift direction can be inverted by (a) inverting the direction of anisotropy (counter- instead of coinjection), (b) by inverting the helicity $\left(I_{p}\right.$ or $B_{t}$ inversion), or (c) by shifting the resonance from the LFS to HFS ( $B_{t}$ variation). In TEXTOR, these predictions could be verified. ${ }^{32,33}$ An example of the spectrum of the highly Doppler shifted energetic ${ }^{3} \mathrm{He}$ and the related NBI and radio-frequency (rf) pulses are shown in Fig. 4.

For "abnormal" $\mathrm{H}$-mode discharge conditions in divertor tokamaks it may not be required to apply such an rf system because the $\tau_{\mathrm{He}}^{*} / \tau_{E}$ helium removal criterion can be met. Nevertheless, the system may be interesting for discharges with a strong internal barrier. Here, the rf may help to push energetic helium ash over the transport barrier.

\section{III.D. Active Control of He and T by Using Ponderomotive Force}

A new method has been proposed to improve the $\mathrm{He}$ exhaust by a pump limiter or a divertor using a resonant rf-ponderomotive force ${ }^{34-37}$ A ponderomotive force is a nonlinear effect of rf-electric fields acting on charged particles, and it is usually large for electrons in a nonresonant term. The ponderomotive force due to perpendicular electric field under the static magnetic field shows the resonance feature of the force acting on the ions. This force can be related to an effective potential that either attracts ions or repulses them depending on the frequency relative to the ion cyclotron frequency. Ions of interest for a fusion reactor are deuterium, tritium, and the helium ash. For the following application the potential distribution of Fig. 5 is proposed. The frequency is 1.1 times the ion cyclotron frequency of tritium.

The proposal has been installed as an rf antenna in front of the scoops of the pump limiter. The potential for incoming $\mathrm{He}^{++}$is negative, and therefore, it can pass the antenna. The $\mathrm{He}^{++}$is guided by the magnetic field to the neutralizer plate where it leaves as neutral helium. With $50 \%$ probability this helium streams toward the pumps and is removed. The rest of the helium diffuses back toward the scoop entrance. In particular at discharges with high electron density, this helium becomes singly ionized. Figure 5 shows a positive potential for the $\mathrm{He}^{+}$ such that it is repelled from the rf antenna back to the neutralizer plate. Here, the cycle restarts, and-depending on the ionization rate inside the scoops-the efficiency for the helium removal is enhanced.

The frequency is selected such that the potential of the ponderomotive antenna is strongly positive also for $\mathrm{T}^{+}$. Therefore, the tritium is already repelled before it enters the scoop and therefore should not be pumped. This should reduce the effort for the cleaning and preparation of the exhaust gas. The active control of He by using ponderomotive force has been tested by measuring the change in the helium flow in the exhaust channel of one of the ALT-II limiter blades. The helium neutrals are injected close to the neutralizer plate in the scoop behind the limiter blade.

The filled circles in Fig. 6 give the helium pressure increase as a function of the line-averaged density for a toroidal magnetic field of $1.9 \mathrm{~T}$. This field corresponds to 

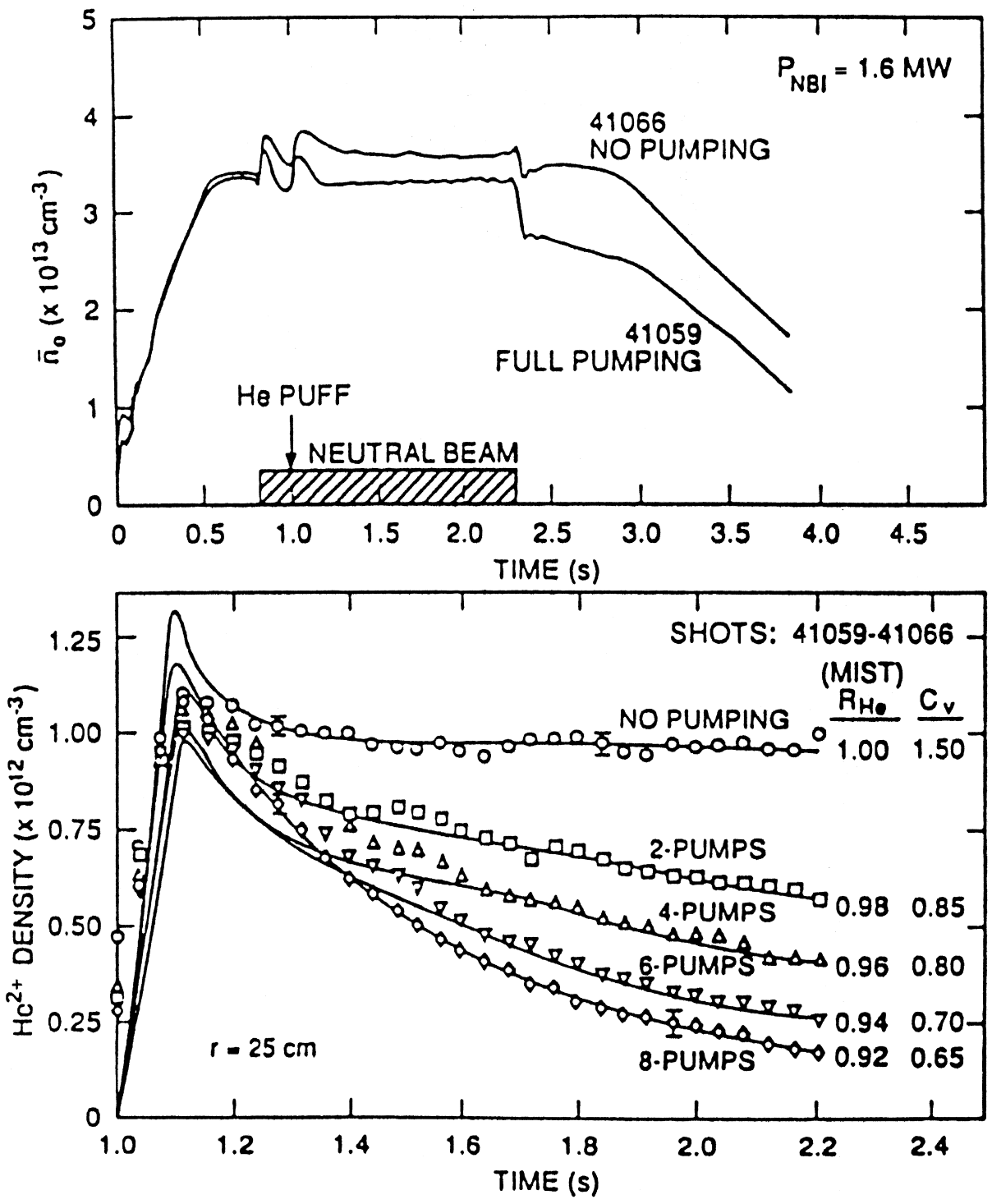

Fig. 3. Line-averaged electron density $n_{e}$ and $\mathrm{He}^{2+}$ density measured with charge-exchange recombination spectroscopy versus time during plasma discharges after a short He puff at $1.0 \mathrm{~s}$ with a different number of pumps active. Solid lines from MIST transport calculations.

a local magnetic field at the radial location of the $\mathrm{rf}$ antenna of $1.6 \mathrm{~T}$, where the resonance frequency is $\sim 1.35$ times higher than the angular ion cyclotron frequency. At this magnetic field a change of the pressure ratios could be detected at a much lower line-averaged density of $1 \times$ $10^{13} \mathrm{~cm}^{-3}$. The highest increase at this magnetic field is $20 \%$ at a line-averaged density of $4.2 \times 10^{13} \mathrm{~cm}^{-3}$. The squares correspond to a $B_{t}$ of $2.25 \mathrm{~T}$ and show a smaller effect than near the resonance.

\section{III.E. He Self-Pumping by Nickel}

A proof of the principle experiment to demonstrate helium self-pumping by nickel ${ }^{38-40}$ has been undertaken in TEXTOR. The experiment used the ALT-I limiter head fitted with two nickel-coated $0.2 \times 0.2 \mathrm{~m}$ INCONEL $^{\circledR}$ plates. Both plates had heaters to maintain the plate temperature at $\sim 350^{\circ} \mathrm{C}$ so that $\mathrm{H}$ or $\mathrm{D}$ would readily diffuse but He would remain trapped. One plate intercepted ion and electron fluxes, and the other received only neutral fluxes. A fresh layer of 5-nm nickel was deposited on the plates before a shot. Two questions addressed in the experiment were (a) would He implanted very near to the surface, i.e., with sheath acceleration from relatively low edge temperatures of 25 to $35 \mathrm{eV}$, remain trapped, and (b) would there be significant trapping of reflected He neutrals. The trapping of helium $(\sim 10 \%$ concentration in a deuterium discharge) and the detrapping of 


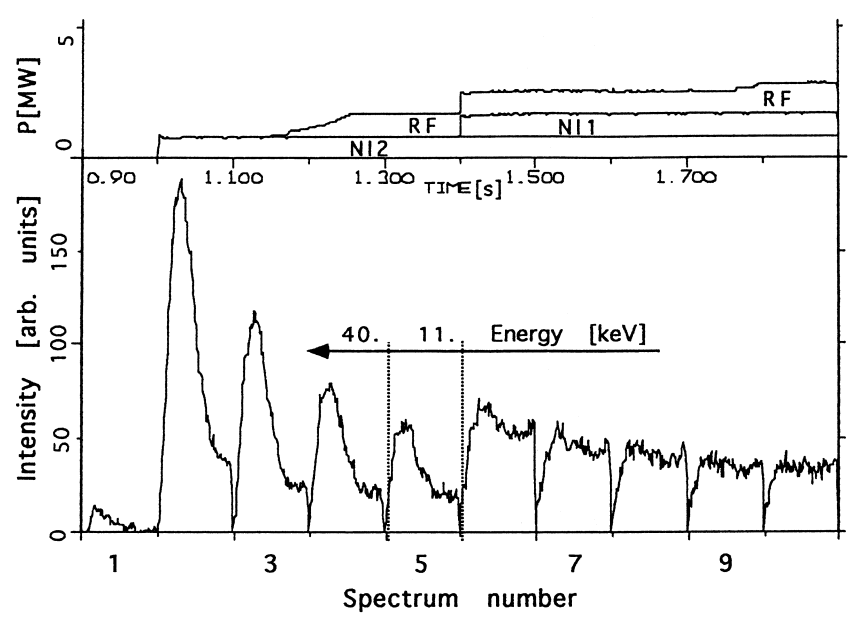

Fig. 4. Shot 54856 with $B_{t}=1.9$ T. Bottom curve: temporal sequence of spectra recorded by an OMA system; line of sight $8 \mathrm{~cm}$ outboard of the magnetic axis. Each spectrum is sampled over a time interval of $100 \mathrm{~ms}$. Top curves: sequence of NBI and rf pulses.

hydrogen isotopes has been studied for different ion fluences and surface temperatures. The results were favorable based on shot-to-shot comparisons of He content in the plasma with and without Ni deposition on the plates and on posttest analysis of the trapped He in the plates. The latter measurement of a trapped He areal density of

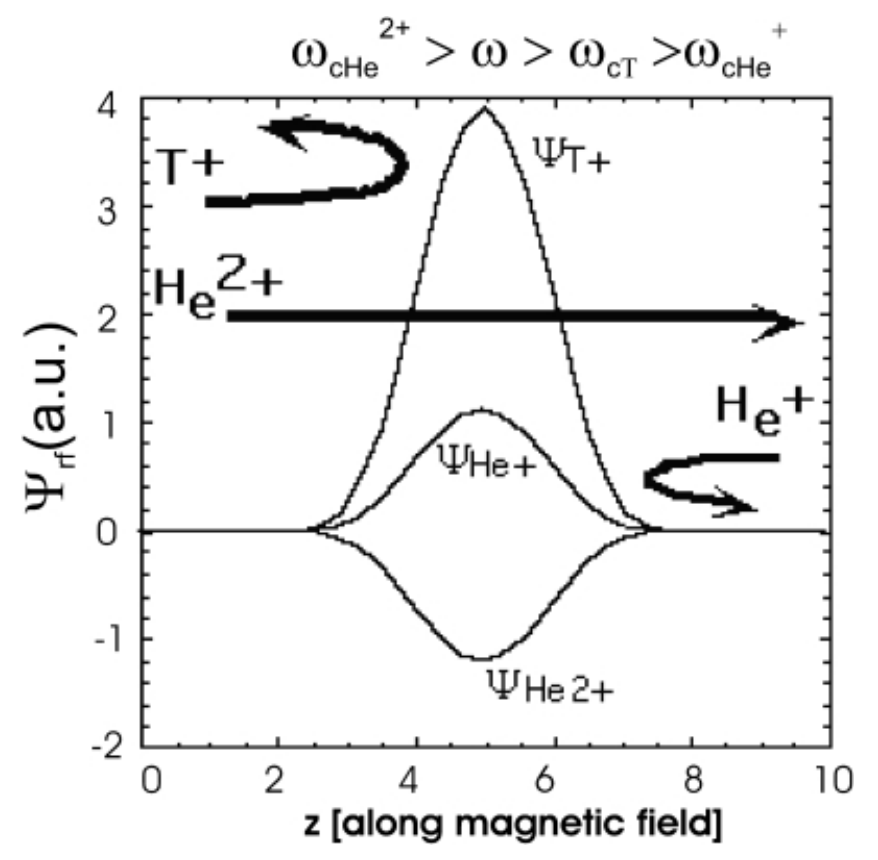

Fig. 5. Concept of improving helium removal and impeding tritium pumping by the application of a ponderomotive force at $\omega=1.1 \cdot \omega_{c i-T}$.

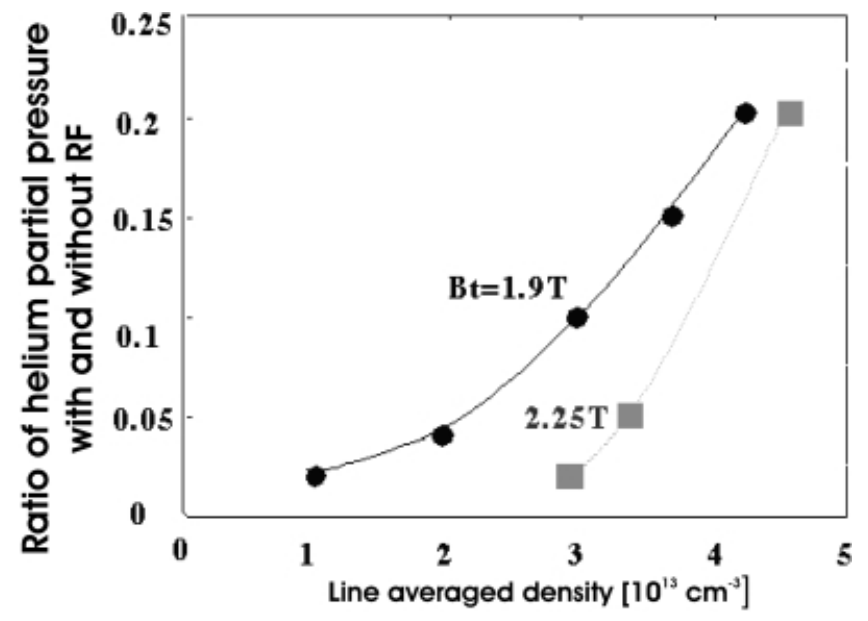

Fig. 6. Increase in the partial helium pressure during application of $\mathrm{rf}$ as a function of the line-averaged density at two magnetic fields. The rf helium filter effect increases with increasing density. $F=8 \mathrm{MHz}, \operatorname{Prf}=$ $3.5 \mathrm{~kW}$.

$\sim 2 \times 10^{20} \mathrm{He} / \mathrm{m}^{2}$ from a series of 21 shots with $\mathrm{Ni}$ coatings of the plates was consistent with expectations based on modeling of the implantation and saturation and ion beam experiments. Trapped helium was found on the trapping plate exposed to only neutral flux as well as the one exposed to He ions. The module was found compatible with overall tokamak operation with essentially no sputtered nickel entering the core plasma. Post tokamak test examination of the trapping plates shows helium atomic concentrations in the deposited nickel consistent with the observed helium removal.

\section{III.F. The Modified Penning Tube for Helium Detection}

In order to measure the gaseous helium partial pressure in the exhaust, a new technique had to be developed. Because the masses of $\mathrm{He}$ and $\mathrm{D}_{2}$ are very close together, methods based on mass spectroscopy are very difficult to apply. Therefore, we have applied a spectroscopic method using a standard Penning tube such as it is used for vacuum pressure measurements. The Penning tube has the major advantage that it excites spectral lines without using filaments that produce disturbing background light as, e.g., ionization gauges would do. The first generation of Penning detectors was equipped with interference filters and photo multipliers ${ }^{41}$ while later versions used a spectrometer plus a charge-coupled device camera. ${ }^{42,43}$ The Penning discharges are robust and operate reliably; because of the intrinsic magnetic field, they are practically insensitive to the magnetic field of the discharge. An example of the Penning calibration curve is shown in Fig. 7. The Penning has meanwhile become a standard system worldwide. 


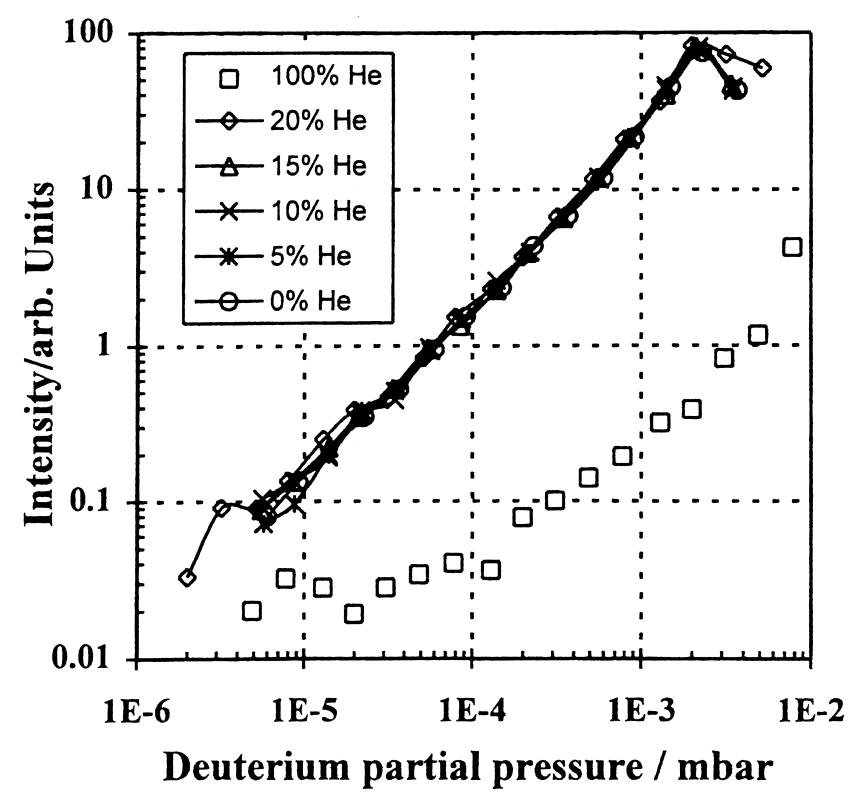

Fig. 7. Intensity of the He line versus $\mathrm{He}$ partial pressure at different $\mathrm{He}$ concentrations. The $0 \% \mathrm{He}$ points are versus total approximate deuterium pressure and show the noise due to the molecular spectrum of deuterium.

\section{PARTICLE REMOVAL}

The ALT-II limiter scoops were equipped with an array of diagnostic tools to study plasma physics and plasma-neutral interactions in the SOL. Most scoops were oriented to collect plasma in both the ion and electron flow directions while others were oriented only in the ion or electron flow direction. Extensive studies of boundary layer asymmetries were carried out in the top/bottom scoop geometries. Outside-facing scoops (facing iondrift direction) received higher flux than inside-facing scoops. Each scoop contained at least one Langmuir probe swept at 100-ms intervals to measure plasma density and temperature. Typical values in an ohmic discharge near the neutralizer plates were $n_{e}=1 \times 10^{12} \mathrm{~cm}^{-3}, T_{e}=10$ to $20 \mathrm{eV}$. In one scoop, a poloidal array of probes measured the distribution across the neutralizer plate (small effect).

Another radial array measured electron-folding lengths (typically 1 to $2 \mathrm{~cm}$ for most parameters). Two particle collection scoops were equipped with additional probes at the entrance of the scoop to study parameter gradients along flux tubes. This arrangement provided valuable information on recycling rates. ${ }^{44}$ One "long" scoop was installed to reduce neutral backflow and investigate pumping efficiency as a function of scoop conductance. An additional fast-scanning Langmuir probe was used to measure SOL decay profiles above and below the limiter belt with data correlated to measurements in the particle exhaust scoops. The magnetic connection length of the flux tube was sufficiently long to decouple fast-scanning probe measurements from the limiter belt. Power deposition studies were carried out via thermocouples embedded in the neutralizer plates and infrared imaging of the limiter blade. Power fluxes up to three times higher were observed on the ion-drift side of the limiter. Plasma conditions at the neutralizer plates, neutral gas migration, and net exhaust efficiency were studied as functions of $q(r), I_{\text {total }}$, gas puff sequences, and line density through the plasma core. Gas pressures near the neutralizer plates were estimated to be in the 1- to 5 -mtorr range. Pressure buildup in the pump station plenum boxes was observed to increase nonlinearly with discharge line density. Ten percent exhaust efficiency required removal rates on the order of 1 to $3 \times 10^{20}$ particles/s or $\sim 2$ torr $\ell / / \mathrm{s}_{2}$ by the ALT-II pumps. For standard ohmic discharges these conditions could be achieved with all eight pump stations active.

\section{IV.A. Recycling and Particle Exhaust}

Particle exhaust studies have been carried out under ohmic heating conditions as well as with NBI and ion cyclotron resonance auxiliary heating. ${ }^{45}$ Quantitative measurements of $\mathrm{D}_{\alpha}$ emission have been used to determine the particle efflux from the plasma. Roughly one-third of the $\mathrm{D}_{\alpha}$ emission occurs in a diffuse halo around the belt limiter. The particle confinement time is less than the energy confinement time by a factor of typically 4 . The modeled fluxes agree with the measured ones well within a factor of 2 (Ref. 46). The particle efflux and the pumped flux both increase with density as well as with NBI heating power. The measured exhaust efficiency has been found to be $\sim 2 \%$, with the highest values observed in high-density NBI discharges. Calculations of ignition requirements in fusion reactors with profile effects included have been made, with results indicating that $2 \%$ exhaust efficiency is sufficient for a reactor. ${ }^{44}$ We tentatively attribute the difference of the measured exhaust efficiencies of helium and deuterium in the different local recycling paths and ionization characteristics of the two species.

Plasma and neutral flows in the ALT-II scoops have been simulated. The scoop may be viewed as a nonlinear amplifier of the plasma particle flux; the amplification is found to range from about two to three for most cases. A schematic view of the scoop is shown in Fig. 8. Flow reversal in the scoop ${ }^{47}$ is found in some NBI cases and in particular in the highest density case. The flux amplification and flow reversal have been studied by injecting helium into the scoop of ALT-II and by investigating the recycling. As shown in Fig. 9, the recycling coefficient starts to increase at a throat electron density higher than $1 \times 10^{19} \mathrm{~m}^{-3}$.

The transport into the SOL also has been extensively investigated. The investigations are essential for the question of whether the transport in the plasma edge is neoclassical or anomalous. The measurements were 


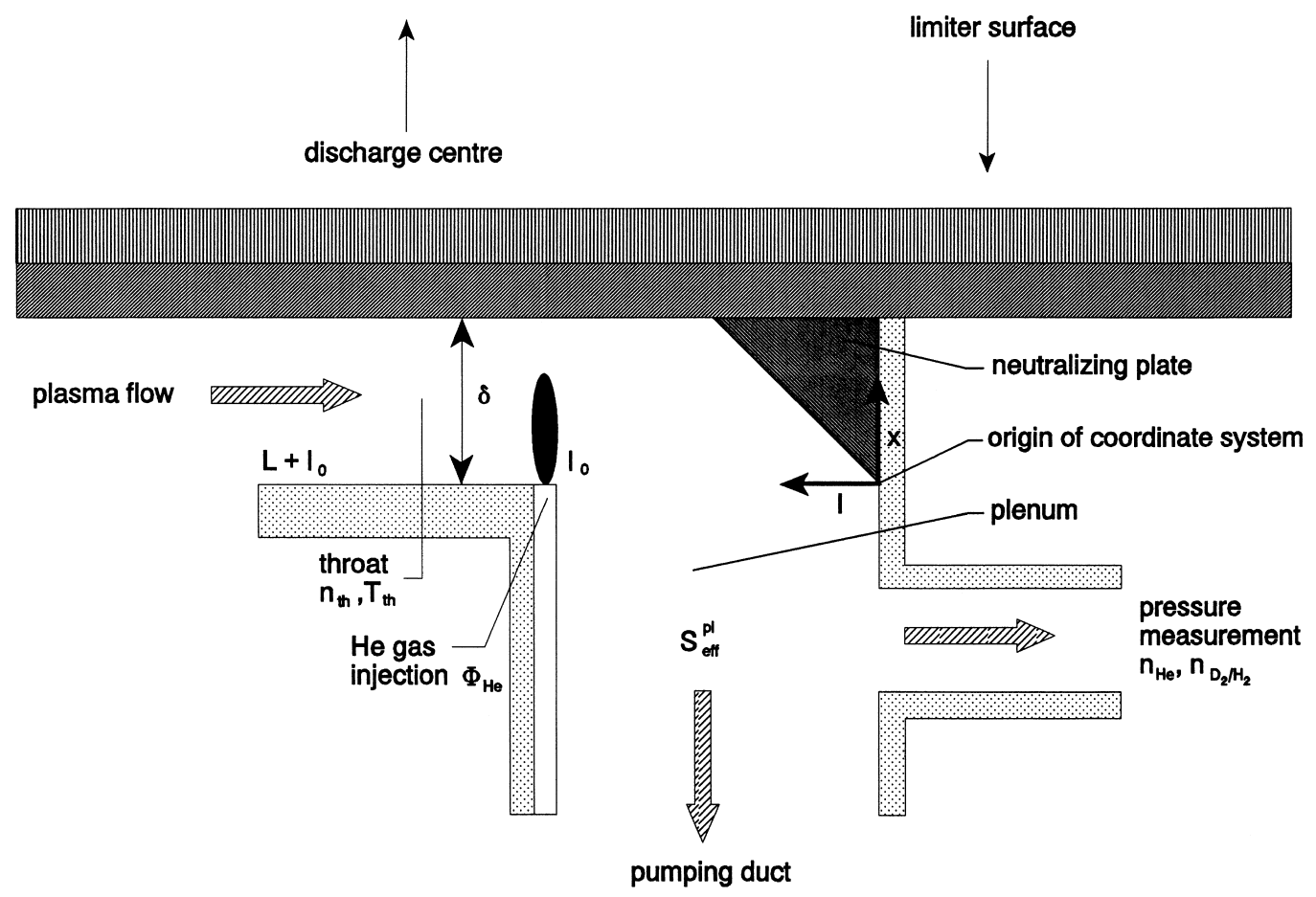

Fig. 8. Schematic description of the throat of ALT-II blade No. 6; the width is $0.027 \mathrm{~m}$, the length $L$ in the toroidal direction is $0.18 \mathrm{~m}$, and the height $h$ is $0.135 \mathrm{~m}$. The origin of the coordinate system used is at the neutralizer plate. The helium gas flow $\left(I_{a}\right)$ is injected into the throat. The measured effective pumping speed at the plenum amounts to $S=650 \ell / \mathrm{s}$. The partial densities of helium and deuterium are determined at the beginning of the pumping duct.

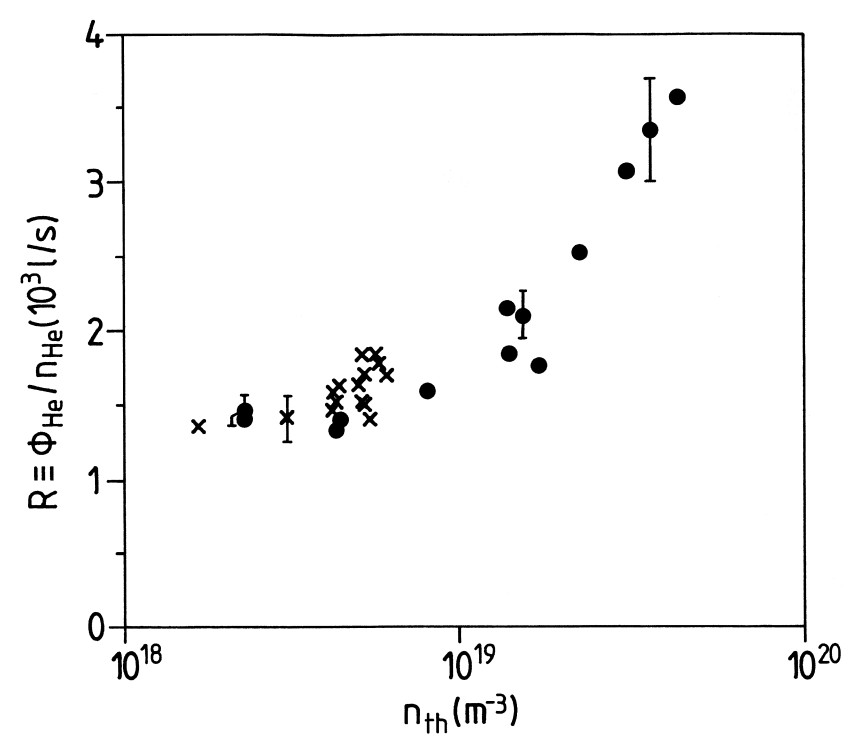

Fig. 9. Dependence of the helium flow into the throat normalized to the partial helium density in the plenum. Crosses: ohmic and neutral beam heated discharges with all eight ALT-II blades at $0.46 \mathrm{~m}$ minor radius. Circles: discharges with blade No. 6 inserted to $0.44 \mathrm{~m}$ and the other blades retracted to $r=0.475 \mathrm{~m}$. performed mainly by scanning Langmuir probes, ${ }^{48-50}$ and both low- and high-frequency fluxes were analyzed. The dominance of turbulence-driven fluxes was manifested; however, details depend on the mode of confinement (e.g., RI mode ${ }^{51}$ ) and on the velocity shear. ${ }^{52-54}$

\section{POWER DISTRIBUTION}

Thermographic measurements ${ }^{55-61}$ have been used to determine the temperature rise of the surface of the graphite tiles and to derive the power distribution to the surface. The measurement of the surface temperature is mainly a safety issue, namely to prevent overheating. It has been found that the layout of the graphite tiles always had a sufficiently large safety margin such that under all operating conditions overheating of leading edges was avoided. In addition, it was established that the adjustment options of the blades were perfect such that the end tiles did not receive excessive heating. Even during disruptions, no overheating of tile materials was observed.

The second aim, the determination of radial structure of the energy flowing along the magnetic field lines, is considerably more demanding than the first part. It 
requires solving the heat diffusion equation using the temperature rise as a boundary condition and using the detailed knowledge of the magnetic field lines relative to the ALT position (including field ripple and plasma shifts) as well as the surface normal vector at all positions along the surface. Using all this information, the radial profile of the energy flux is obtained and has the shape of the curve given in Fig. 10, namely a part with a short power decay length near the point of limiter tangency followed by a longer one radially more outward. The short decay length amounts for all conditions (ohmic, NBI heated, ion cyclotron resonance heated, reversed plasma current, or $B t$ ) practically always 1.5 to $2.5 \mathrm{~mm}$ while the radially more outward part has a decay length of 6 to $8 \mathrm{~mm}$. As discussed also in the section on disruptions, this structure is even preserved there.

\section{BIASING}

ALT-II is electrically isolated from the liner and the wall; this allows the application of an electrical voltage (biasing) ${ }^{62,63}$ between the belt limiter and the liner or the inner bumper limiter, which is electrically connected with the liner. Essentially, two configurations of the plasma positioning for biasing have been studied: (a) the plasma shifted such that it had equal contact with ALT-II and the inner bumper limiter and (b) the plasma touching ALT-II and radially well separated from the bumper. In case (a) an electric field is established along the magnetic field lines causing a large electrical current (500 A given as maximum of the power supply) flowing between both limiters. This mode of operation showed no remarkable effect on the plasma. With configuration (b) a radial elec-

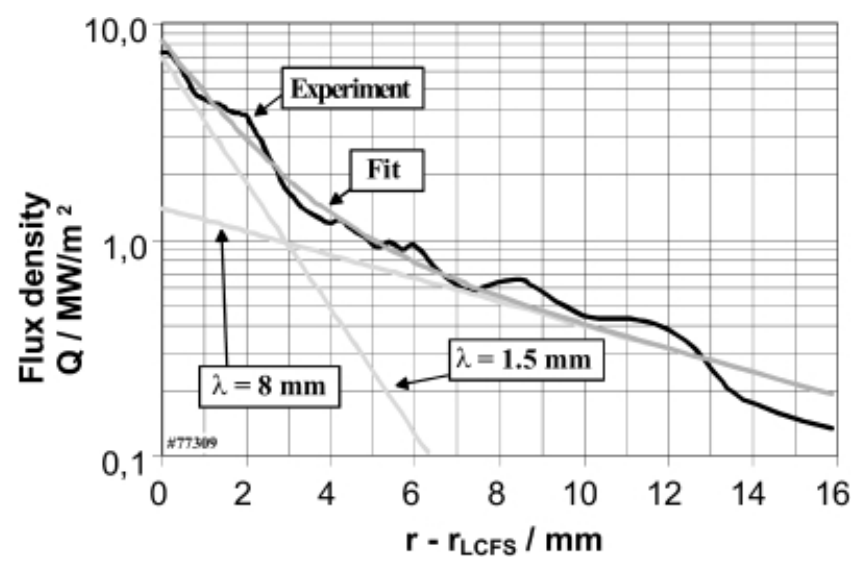

Fig. 10. Structure of the energy in the SOL flowing along magnetic field lines. The flux density scale (vertical axis) is logarithmic, and the horizontal axis is the radius in millimeters measured from the last closed flux surface. tric field was created with the potential dropping nearly exclusively in the SOL; this means that the plasma potential is determined by the ALT-II limiter. In this mode, the recycling and edge density are modified without affecting the core confinement. The strongest effect is a modification of the drift in the SOL due to the modified $\mathrm{E} \times \mathrm{B}$. The biasing has been utilized to guide particles more efficiently into the ALT-II exhaust scoop and thereby enhance the particle removal.

\section{PROTECTING LAYER}

The ALT-II system is equipped with several independent gas injection lines. One set of the gas lines leads to the toroidal leading edge of ALT-II ending there in ten poloidally distributed outlet holes. This gas line has been utilized to test the concept of protecting a strongly exposed surface by deposition of a fresh surface layer. For this reason, the particular blade with the gas line was radially moved into the plasma such that the leading edge showed a strong carbon erosion. Then, in a following set of discharges, silane gas ${ }^{64}$ was blown to the leading edge. As expected, the silane covered the exposed surface and reduced the excessive carbon erosion.

\section{MODES OF IMPROVED CONFINEMENT}

\section{VIII.A. RI Mode}

The presence of a pump limiter in TEXTOR did not lead immediately to an improved confinement such as the H-mode in divertor experiments. However, the controlled addition of the seed impurity neon led to the RI mode ${ }^{65}$ with very good confinement properties, in particular, close to Greenwald limit. For achieving and controlling the RI mode, the "sink" of the neon impurities by the ALT-II pumping was an essential ingredient. Most of the work on the RI mode is described in a separate section and can be omitted here. One should only mention a further energy confinement improvement by a slow and continuous gas blowing ${ }^{66}$ through the pipes at the ALT-II blades.

\section{VIII.B. Pellet Injection}

Pellet injection is a means of deep fueling of the plasma discharge. Pellet injection is applied on one hand for obtaining an improved fueling efficiency and an improved confinement state, but on the other hand-since the pellet injection is a major disturbance-for the analysis of the internal plasma state. This disturbance excites characteristic oscillations in the plasma column and may lead to "snakes" 67 or striations, ${ }^{68-70}$ which are often assumed to be associated with rational flux surfaces. Therefore, pellets have been applied to derive the $q(r)$ profile. 
The improvement of a discharge is generally better at higher densities than at lower ones. It is normally observed in tokamaks that the good confinement is inhibited when auxiliary heating is applied. However, during the RI mode with NBI heating and neon-seed impurity control, pellet injection ${ }^{71}$ can lead to a further increase of density and stored energy as shown in Fig. 11.

\section{TRITIUM}

The standard working gas of TEXTOR is deuterium, and deuterium is also used for NBI. Trace amounts of tritium are thus only generated by beam particle interaction. Applicability of a newly developed $\beta$-ray-induced X-ray spectrometry ${ }^{72,73}$ (BIXS) has been examined to measure nondestructively tritium retained on/in the graphite samples.

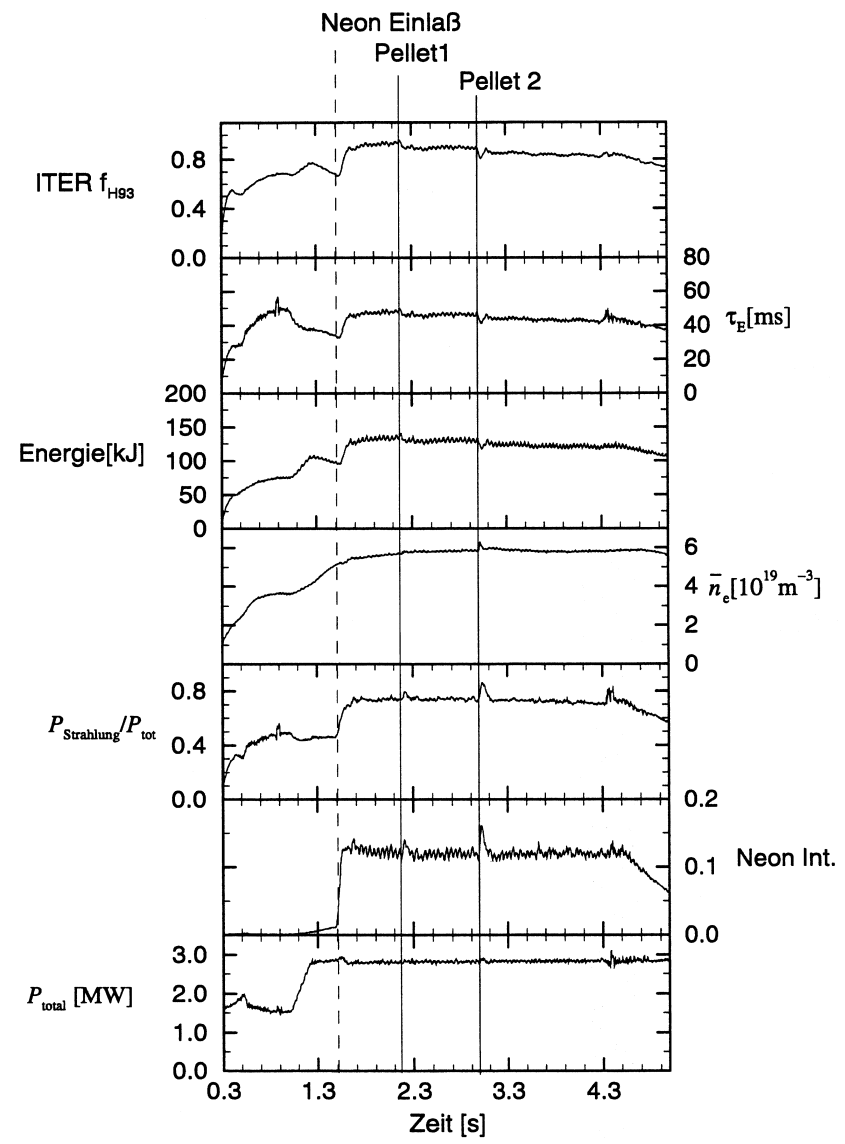

Fig. 11. Example of pellet fueling into an RI-mode discharge. The following traces from bottom to top are shown as function of time: total heating power $P_{t o t}$, brilliance of $\mathrm{Ne}$ VIII line, radiated power fraction $\gamma$, line-averaged density $n_{e}$, diamagnetic energy $E_{d i a}$, energy confinement time $\tau_{e}, f_{\mathrm{H} 93}$, neutron yield $Y$, and $Z_{\text {eff }}$.
Examination was carried out by using the graphite plates irradiated with tritium ions and an ALT-II limiter tile exposed to D plasmas in TEXTOR as shown in Fig. 12. For the former samples, a sharp intense peak and a broad weak peak appeared clearly in the spectra; the former peak was attributed to the characteristic $X$ rays from argon used as a working gas, and the latter peak was assigned to the bremsstrahlung $X$ rays from subsurface layers of graphite. On the other hand, for the latter sample, a rather weak characteristic X-ray peak was observed along with a diminutive bremsstrahlung X-ray
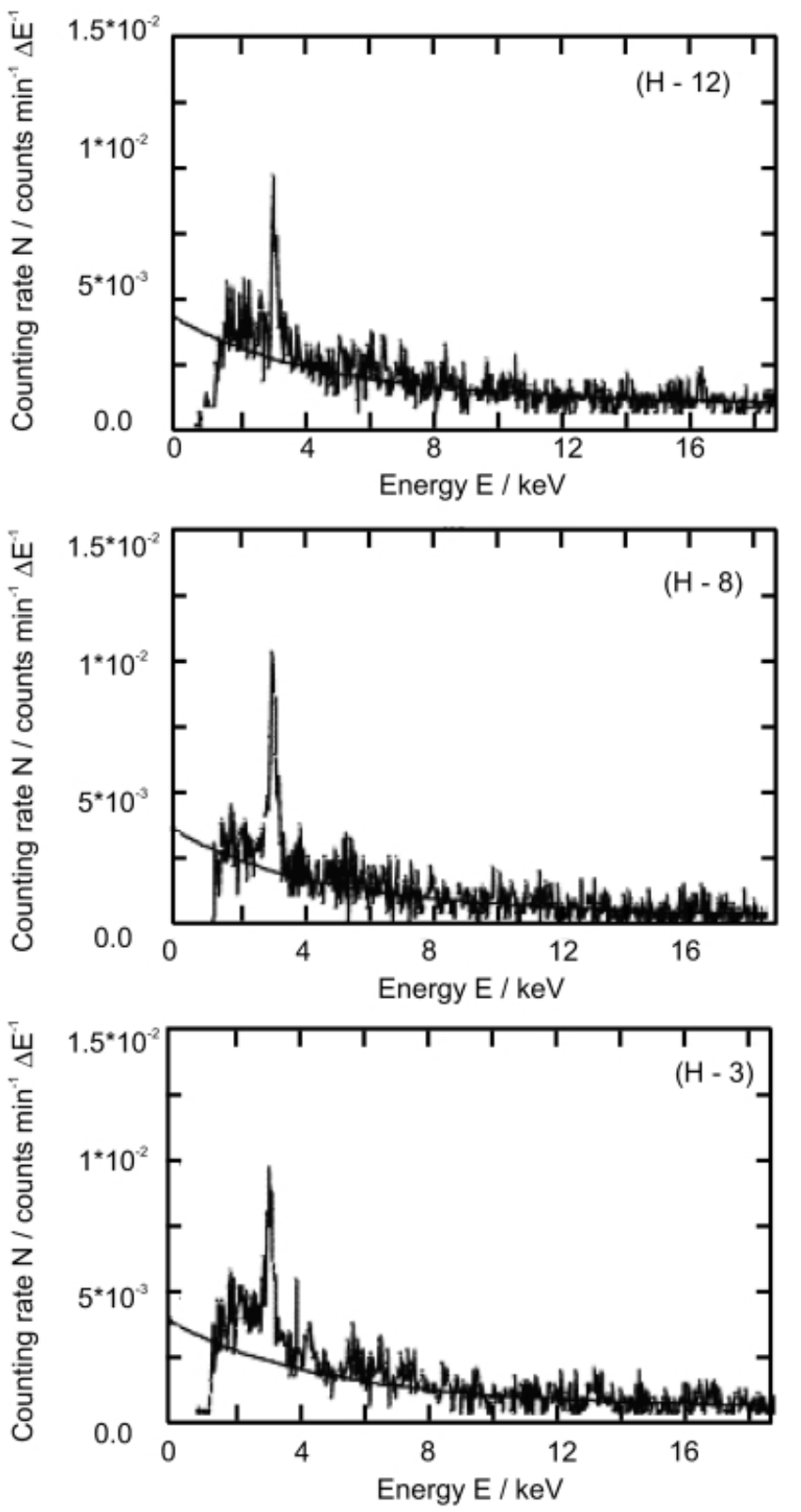

Fig. 12. The X-ray spectra for each measuring spot. Solid lines represent a background level measured without a limiter tile. 
peak. Although the intensities of those $\mathrm{X}$ rays differed from spot to spot, the tritium levels retained on the limiter tile were determined to be 58 to $132 \mathrm{~Bq} / \mathrm{cm}^{2}$. It was concluded, therefore, that valuable information on the amount and the distribution of tritium retained on/in the wall materials can be nondestructively obtained by using the BIXS.

\section{CONCLUSIONS}

The paper has shown the richness of results focused around the international collaboration within the IEA collaboration on pump limiter ALT-II on TEXTOR. Over many years, the collaboration has been very intense and fruitful for all partners. The center of attention was the question of particle and power removal from a tokamak and fusion relevant conditions. A highlight was questions of the helium ash exhaust. On helium removal, basic considerations were carried out on the maximum helium residence time in a fusion reactor, and many innovative treatments and techniques on helium removal were developed. Detailed studies have been performed on the recycling of the working gas deuterium, on the exhaust rate and exhaust efficiency, and on the processes in the exhaust scoop. For the measurement of the power removal, infrared thermography was applied, which revealed a rather complex picture of the power decay length pattern. In order to influence the flow pattern in the SOL, an electrical field (biasing) has been applied, which allows for an optimization of the particle exhaust. Finally, the pump limiter ALT-II is the basis for achieving the high confinement mode (RI mode) with controlled neon radiation in the plasma boundary. Pellet injection improved the mode even further.

\section{REFERENCES}

1. A. E. PONTAU et al., Fusion Technol., 6, 384 (1984).

2. K. H. DIPPEL et al., Proc. 10th Int. Conf. Plasma Physics and Controlled Nuclear Fusion Research, London, England, September 12-19, 1984, Vol. 1, p. 249, International Atomic Energy Agency, Vienna, Austria (1985).

3. K. H. FINKEN, in Tokamak Start-Up, p. 309, H. KNOEPFEL, Ed., Plenum Publishing Corporation (1986).

4. K. H. FINKEN et al., J. Nucl. Mater., 145-147, 825 (1987).

5. D. M. GOEBEL et al., Plasma Phys. Control. Fusion, 29, 473 (1987).

6. G. J. THOMAS et al., J. Nucl. Mater., 145-147, 830 (1987).

7. K. H. DIPPEL et al., J. Nucl. Mater., 145-147, 3 (1987).

8. R. W. CONN et al., Proc. 11th Int. Conf. Plasma Physics and Controlled Nuclear Fusion Research, Kyoto, Japan, November 12-19, 1986, Vol. 1, p. 249, International Atomic Energy Agency, Vienna, Austria (1987).

9. A. HARDTKE et al., J. Nucl. Mater., 162-164, 661 (1989).
10. R. T. McGRATH et al., J. Nucl. Mater., 145-147, 660 (1987).

11. D. M. GOEBEL et al., J. Nucl. Mater., 162-164, 115 (1989).

12. W. J. CORBETT et al., J. Nucl. Mater., 162-164, 221 (1989).

13. K. H. DIPPEL et al., Proc. 12th Int. Conf. Plasma Physics and Controlled Nuclear Fusion Research, Vol. 1, p. 453, International Atomic Energy Agency, Vienna, Austria (1989).

14. W. J. CORBETT et al., J. Vac. Sci. Technol. A, 3, 1772 (1990).

15. D. S. GRAY et al., J. Nucl. Mater., 176-177, 810 (1990).

16. W. J. CORBETT et al., Nucl. Fusion, 31, 1067 (1991).

17. D. S. GRAY et al., J. Nucl. Mater., 196-198, 1096 (1992).

18. J. A. BOEDO et al., J. Nucl. Mater., 196-198, 489 (1992).

19. T. BANNO et al., J. Nucl. Mater., 220-222, 478 (1995).

20. R. P. DOERNER et al., J. Nucl. Mater., 176-177, 635 (1990).

21. D. REITER, G. H. WOLF, and H. KEVER, Nucl. Fusion, 30, 2141 (1990).

22. E. REBHAN, U. VIETH, D. REITER, and G. H. WOLF, Nucl. Fusion, 36, 264 (1996).

23. K. H. FINKEN et al., J. Nucl. Mater., 176-177, 816 (1990).

24. D. L. HILLIS et al., Proc. 13th Int. Conf. Plasma Physics and Controlled Fusion Research, IAEA-CN-53/G-II-8, Part III, p. 597, International Atomic Energy Agency, Vienna, Austria (1990).

25. D. L. HILliS et al., Phys. Rev. Lett., 65, 2382 (1990).

26. K. H. FINKEN et al., in Contributions to High-Temperature Plasma Physics, p. 390, K. H. SPATSCHEK and J. UHLENBUSCH, Eds., Akademie Verlag, Berlin, Germany (1994).

27. U. SAMM et al., J. Nucl. Mater., 196-198, 633 (1992).

28. D. L. HILLIS et al., J. Nucl. Mater., 196-198, 35 (1992).

29. C. S. CHANG and P. COLESTOCK, Phys. Fluids B, 2, 310 (1990).

30. C.S. CHANG, Phys. Fluids B, 3, 259 (1991).

31. C. S. CHANG, J. Y. LEE, and H. WEITZNER, Phys. Fluids B, 3, 3429 (1991).

32. K. H. FINKEN et al., Phys. Rev. Lett., 73, 436 (1994).

33. R. KOCH et al., Plasma Phys. Control. Fusion, 37, Suppl. 11A, A291 (1995).

34. T. SHOJI, Y. SAKAWA, K. TSUJII, T. WATARI, and K. H. FINKEN, J. Nucl. Mater., 220-222, 483 (1995).

35. T. SHOJI et al., J. Nucl. Mater., 313-316, 1262 (2003).

36. G. MANK, T. SHOJI, K. H. FINKEN, and Y. SAKAWA, Czech. J. Phys., 49, 299 (1999).

37. T. SHOJI and Y. SAKAWA, J. Plasma Fusion Res., 77, 111 (2001).

38. J. N. BROOKS et al., J. Nucl. Mater., 176-177, 640 (1990).

39. J. N. BROOKS et al., J. Nucl. Mater., 196-198, 664 (1992).

40. R. E. NYGREN et al., J. Nucl. Mater., 196-198, 558 (1992).

41. K. H. FINKEN, K. H. DIPPEL, W. Y. BAEK, and A. HARDTKE, Rev. Sci. Instrum., 63, 1 (1992).

42. T. DENNER, K. H. FINKEN, and G. MANK, Rev. Sci. Instrum., 67, 3515 (1996).

43. D. L. HILLIS et al., Fusion Eng. Des., 34-35, 347 (1997).

44. D. S. GRAY, J. A. BOEDO, and R. W. CONN, Nucl. Fusion, 37, 1437 (1997).

45. D. S. GRAY et al., Nucl. Fusion, 38, 1585 (1998).

46. D. S. GRAY et al., Physics of Plasmas, 6, 2816 (1999). 


\section{Finken et al. TEXTOR TOROIDAL PUMP LIMITER ALT-II}

47. G. MANK et al., Nucl. Fusion, 34, 1641 (1994).

48. J. BOEDO et al., Rev. Sci. Instrum., 69, 2663 (1998).

49. J. A. BOEDO et al., Rev. Sci. Instrum., 70, 2997 (1999).

50. J. BOEDO et al., Rev. Sci. Instrum., 72, 1379 (2001).

51. J. A. BOEDO et al., Nucl. Fusion, 40, 209 (2000).

52. J. BOEDO et al., Nucl. Fusion, 40, 1397 (2000).

53. J. A. BOEDO et al., Phys. Rev. Lett., 84, 2630 (2000).

54. J. A. BOEDO et al., Nucl. Fusion, 42, 117 (2002).

55. K. H. FINKEN et al., J. Nucl. Mater., 162-164, 655 (1989).

56. K. H. FINKEN et al., Nucl. Fusion, 32, 915 (1992).

57. K. H. FINKEN et al., J. Nucl. Mater., 196-198, 220 (1992).

58. A. V. NEDOSPASOV et al., J. Nucl. Mater., 196-198, 676 (1992).

59. T. DENNER, K. H. FINKEN, G. MANK, and N. NODA, Nucl. Fusion, 39, 83 (1999).

60. K. H. FINKEN, T. DENNER, and G. MANK, Nucl. Fusion, 40, 339 (2000).

61. K. H. FINKEN, A. KRAEMER-FLECKEN, G. MANK, and S. S. ABDULLAEV, J. Nucl. Mater., 290-293, 1064 (2001).
62. R. P. DOERNER et al., Nucl. Fusion, 34, 975 (1994).

63. E. GRAVIER et al., Nucl. Fusion, 42, 653 (2002).

64. G. MANK et al., J. Nucl. Mater., 241-234, 821 (1997).

65. A. M. MESSIAEN et al., Phys. Rev. Lett., 77, 2487 (1996).

66. G. MANK et al., Phys. Rev. Lett., 85, 2312 (2000).

67. K. H. FINKEN et al., AIP Conference Proceedings 345, Int. Conf. Plasma Physics ICPP, p. 172, American Institute of Physics, Woodbury, New York (1994).

68. K. H. FINKEN and W. O. HOFER, in Physical Processes of the Interaction of Fusion Plasmas with Solids, W. O. HOFER and J. ROTH, Eds., Series: Plasma-Materials Interactions, Academic Press, New York (1996).

69. K. N.SATO, Trans. IEE Japan, 113-A, 801 (1993).

70. K. H. FINKEN et al., Plasma Phys. Control. Fusion, 39, A351 (1997).

71. J. HOBIRK et al., Nucl. Fusion, 40, 1469 (2000).

72. M. MATSUYAMA et al., J. Nucl. Mater., 290-293, 437 (2001).

73. K. MIYASAKA et al., J. Nucl. Mater., 290-293, 448 (2001). 